\title{
Association of PNPLA3 I148M Variant With Chronic Viral Hepatitis, Autoimmune Liver Diseases and Outcomes of Liver Transplantation
}

\author{
Ning Geng ${ }^{1}$; Yong-Ning Xin ${ }^{2, *}$; Harry Hua-Xiang Xia ${ }^{3}$; Man Jiang ${ }^{2}$; Jian Wang ${ }^{1}$; Yang Liu ${ }^{1}$; \\ Li-Zhen Chen ${ }^{1}$; Shi-Ying Xuan ${ }^{2,}$ \\ ${ }^{1}$ Qingdao Municipal Hospital, Medical College, Qingdao University, Qingdao, P. R. China \\ 2 Department of Gastroenterology, Qingdao Municipal Hospital, Qingdao, P. R. China \\ ${ }^{3}$ Qingdao Municipal Hospital, Qingdao University, Qingdao, P. R. China \\ ${ }^{*}$ Corresponding Authors: Yong-Ning Xin, Department of Gastroenterology, Qingdao Municipal Hospital, P. O. Box: 266021, Qingdao, P. R. China. E-mail: xinyongning@gmail.com; Shi-Ying \\ Xuan, Department of Gastroenterology, Qingdao Municipal Hospital, P. O. Box: 266021, Qingdao, P. R. China. Tel: +86-53288905508, Fax:+86-53282031522, E-mail:dxyxyn@gmail.com
}

Received: December 29, 2014; Revised: February 15, 2015; Accepted: March 1, 2015

\begin{abstract}
Context: The PNPLA3 I148M variant has been recognized as a genetic determinant of liver fat content and a genetic risk factor of liver damage progression associated with steatohepatitis. The I148M variant is associated with many chronic liver diseases. However, its potential association with inflammatory and autoimmune liver diseases has not been established.

Evidence Acquisition: We systemically reviewed the potential associations of I148M variant with chronic viral hepatitis, autoimmune liver diseases and the outcome of liver transplantation, explored the underlying molecular mechanisms and tried to translate them into more individualized decision-making and personalized medicine.

Results: There were associations between I148M variant and chronic viral hepatitis and autoimmune liver diseases and differential associations of $1148 \mathrm{M}$ variant in donors and recipients with post-liver transplant outcomes. 1148M variant may activate the development of steatosis caused by host metabolic disorders in chronic viral hepatitis, but few researches were found to illustrate the mechanisms in autoimmune liver diseases. The peripherally mediated mechanism (via extrahepatic adipose tissue) may play a principal role in triglyceride accumulation regardless of adiponutrin activity in the graft liver.

Conclusions: Evidences have shown the associations between I148M variant and mentioned diseases. I148M variant induced steatosis may be involved in the mechanism of chronic viral hepatitis and genetic considered personalized therapies, especially for PSC male patients. It is also crucial to pay attention to this parameter in donor selection and prognosis estimation in liver transplantation.
\end{abstract}

Keywords: PNPLA3; Polymorphism; Hepatitis B, Chronic; Hepatitis C, Chronic; Autoimmune Hepatitis; Liver Transplantation

\section{Context}

In 2008, a Single Nucleotide Polymorphism (SNP) in the patatin-like phospholipase domain-containing 3 (PNPLA3, adiponutrin) gene or rs738409 polymorphism was reported to be significantly associated with liver fat content (1). This SNP represents a substitution from cytosine to guanine, resulting in a switch from isoleucine to methionine at residue 148 (I148M). Since then, extensive investigation on the association between PNPLA3 I148M or rs738409 polymorphism and liver diseases has been performed. Various studies revealed that the PNPLA3 I148M variant is associated with development and progression of liver disease, including non-alcoholic fatty liver disease (NAFLD) (2-4), alcoholic fatty liver disease (5) as well as susceptibility to hepatocellular carcinoma (HCC) $(6,7)$. However, its association with other inflammatory and autoimmune liver diseases has not been fully elucidated. Whether there is an association between the PNPLA3 I148M variant and chronic viral hepatitis and autoimmune liver diseases, and whether PNPLA3 I148M variant plays a role in liver transplantation has not been es- tablished, although some studies addressed these issues and demonstrated potential associations. Moreover, the mechanisms by which PNPLA3 I148M variant may be involved in development and progression of these diseases are largely unclear. Therefore, the aim of this article was to systematically review the current knowledge on the association between PNPLA3 I148M variant and chronic viral hepatitis and autoimmune liver diseases and its possible role in liver transplantation. The aim was also to explore underlying molecular mechanisms and translate them into more individualized decision-making and personalized medicine.

\section{Evidence Acquisition}

We systemically reviewed potential associations of I148M variant with chronic viral hepatitis, autoimmune liver diseases and the outcome of liver transplantation. All references were extracted from sources such as PubMed, EMBASE and other databases with keywords

Copyright (C) 2015, Kowsar Corp. This is an open-access article distributed under the terms of the Creative Commons Attribution-NonCommercial 4.0 International License (http://creativecommons.org/licenses/by-nc/4.0/) which permits copy and redistribute the material just in noncommercial usages, provided the original work is properly cited. 
"PNPLA3 I148M", "hepatitis C", "HCV", "hepatitis B", "HBV", "autoimmune liver diseases", "autoimmune hepatitis", "primary sclerosing cholangitis", "PSC" and "primary biliary cirrhosis", "PBC" and "Liver Transplantation".

\subsection{PNPLA3 I148M Variant and Chronic Viral Hepatitis}

\subsubsection{Association Between I148M Variant and Chronic Hepatitis C}

Hepatic steatosis is a common symptom in patients with chronic hepatitis $\mathrm{C}(\mathrm{CHC})(8)$ and associated with severe fibrosis (9) and a low rate of sustained virological responses (SVR) to antiviral therapy (10). Moreover, steatosis is thought to be a possible risk factor of the development of HCC in hepatitis C virus (HCV) infection $(11,12)$.

In 2011, Valenti et al. reported the first study on the association between PNPLA3 I148M variant and CHC (13). The group analyzed two independent series of 325 and 494 CHC patients in Italy and found that the I148M variant was associated not only with liver steatosis, but also with fibrosis (13). At the same time, Trepo et al. in a study of 537 Caucasian CHC patients in Belgium, Germany and France, observed that PNPLA3 I148M variant favored steatosis and fibrosis progression in $\mathrm{CHC}$ (14). Recent studies indicated that the PNPLA3 I148M variant is a risk factor of fibrosis progression in CHC $(15,16)$. An increased association of the I148M variant with steatosis has been observed, especially in HCV infection with non-3 genotypes $(17,18)$ or in CHC patients with excessive abdominal fat (19). In the study by Zampino et al. (19) the waist circumference, which is highly correlated with visceral adipose tissue in children, adolescents and adults (20), increased the association of the I148M variant with liver steatosis. The group suggested a simple method to modulate the development of liver steatosis in CHC patients, healthy eating and moderate exercise to control body weight.

At present, the influence of PNPLA3 polymorphism on SVR remains controversial. On one hand, Valenti et al. reported that $\mathrm{CHC}$ patients with the I148M variant, who accounted for $10 \%$ of the patients, had a roughly $50 \%$ higher risk of cirrhosis than other PNPLA3 genotypes, and the I148M variant was significantly associated with a lower SVR rate in patients with genotype 1 and $4 \mathrm{HCV}$ infection (13). Moreover, Valenti L et al. (21) reported this association between I148M variant and SVR in patients with genotype 1 and $4 \mathrm{HCV}$ and bridging fibrosis, whereas there was no association in patients with genotypes 1 and $4 \mathrm{HCV}$ without bridging fibrosis or in those with genotypes 2 and $3 \mathrm{HCV}$. On the other hand, Trepo et al. (14) observed no association between PNPLA3 and SVR. Clark and colleagues (22) also did not observe any association between the PNPLA3 I148M variant and SVR, although they further demonstrated the association between the PNPLA3 I148M variant and severe steatosis in patients with previously untreated genotype $1 \mathrm{HCV}$ infection. This finding was confirmed by a recent Japanese study (23). These studies with negative results suggest that metabolic steatosis, rather than genetic steatosis, may be the possible mechanism of low SVR rate during antiviral therapy (24). Therefore, further investigations are required regarding the association between the PNPLA3 I148M variant and SVR to understand the mechanisms for treatment failure in patients undergoing antiviral therapy on the genetic level to improve diagnosis and treatment based on genetic risk stratification.

In their first study of this genetic polymorphism in $\mathrm{CHC}$, Valenti et al. demonstrated an association of the PNPLA3 I148M variant with hepatocarcinogenesis (13), which was confirmed by others $(25,26)$. In 2013, Guyot et al. (27) observed that the PNPLA3 I148M variant was associated with an increased occurrence of HCC in patients with HCV-associated cirrhosis. In 2014, Sato et al. (28) reported that the PNPLA3 I148M variant accelerated hepatocarcinogenesis in $\mathrm{CHC}$, which was determined by genotyping PNPLA3 in 358 hepatitis HCV-associated HCC patients. These findings all indicate the crucial role of the PNPLA3 I148M variant in hepatocarcinogenesis in CHC patients. Thus, taking this genetic factor into consideration would assist in creating a reasonable therapeutic strategy to prevent future development of $\mathrm{HCC}$ in $\mathrm{CHC}$ patients.

\subsubsection{Association Between I148M Variant and Chronic Hepatitis $B$}

In patients with Chronic Hepatitis B (CHB), steatosis also represents a common histopathological feature (29, 30). It is not only associated with severe fibrosis, but also with CHB progression $(30,31)$. Whether host genetic factors contribute to the pathogenesis of steatosis in $\mathrm{CHB}$ remains unknown. Vigano et al. demonstrated that PNPLA3 $148 \mathrm{M}$ polymorphism was an independent predictor of severe steatosis in Italian patients with biopsy-proven-CHB (32). However, this variant was not associated with $\mathrm{CHB}$ or HBV-related cirrhosis $(33,34)$.

\subsubsection{Mechanisms of I148M Variant in the Two Chronic Viral Hepatitis}

It has been postulated that lipid metabolism acts as a crucial factor in steatosis development. Disruption of lipid metabolism in $\mathrm{CHC}$ is reported to be viral genotypespecific (35-37), whereas, viral factors play a predominant role in the development of steatosis (36) in genotype 3 $\mathrm{HCV}$ infection, host metabolic disorders are the major determinants of hepatic steatosis in non-3 genotype HCV infection (37). To understand the possible role of PNPLA3 in viral factor-mediated steatosis, one needs to understand the importance of lipid metabolism in viral packaging. Lipids play a crucial role in the life cycle of HCV (38), for example, HCV escapes immune clearance and establishes persistent infection through host lipid metabolism (39). Thus, HCV has a unique feature; its assembly step is implicated in the host's lipid metabolism (40). It has been demonstrated that intracellular distribution of lipid 
droplets (LDs), which plays an important role in the deposition of triglyceride and cholesterylesters (41), changes profoundly in HCV infection (42). Moreover, virus assembly is drastically impaired when the interaction of HCV core protein with LDs is prevented (43). The PNPLA3 I $148 \mathrm{M}$ variant has been shown to be associated with lipid metabolism in many chronic liver diseases and over $90 \%$ of the PNPLA3 protein are located in LDs in hepatocytes (44). A recent study demonstrated that the I148M variant increased LD development and functions (45), indicating that the I148M variant may play a role in CHC-associated steatosis through this virus-induced pathway (39), although this possible mechanism needs to be further investigated.

The PNPLA3 I148M variant participates in host metabolic disorders as well. The association of the PNPLA3 I148M variant with steatosis or fibrosis is stronger in non-3 genotype HCV infection than in genotype $3 \mathrm{HCV}$ infection, supporting the important role of host metabolic factors in $\mathrm{CHC}$ and $\mathrm{CHB}(17,18,34)$. Recently, Sato et al. (28) suggested that the PNPLA3 I148M variant may be involved in hepatocarcinogenesis by inducing steatosis in the liver in CHC, as the PNPLA3 I148M variant has been identified as a risk factor of steatosis-mediated liver damage and fibrosis progression. Based on available findings, we hypothesize the possible mechanisms by which the PNPLA3 I148M variant plays an important role in the development and progression of liver diseases in chronic hepatitis (Figure 1). As stated above, the presence of steatosis is an essential factor in historic progression of viral hepatitis and viral features, such as genotypes and viral load play no role in the association between PNPLA3 I148M variant and liver disease, indicating that host metabolic disorders rather than viral factors are the leading cause of steatosis in either $\mathrm{CHC}$ or $\mathrm{CHB}(21,32,46)$. Thus, it is hypothesized that viral and host factors are involved in the main mechanisms of development of steatosis in hepatitis and the PNPLA3 I148M variant may participate in the mechanisms (Figure 1). However, further investigation is required regarding the underlying molecular mechanisms by which PNPLA3 I148M polymorphism plays an important role in viral hepatitis and development and progression of liver diseases such as steatosis, fibrosis, cirrhosis and HCC in chronic hepatitis to confirm the proposed hypothesis.

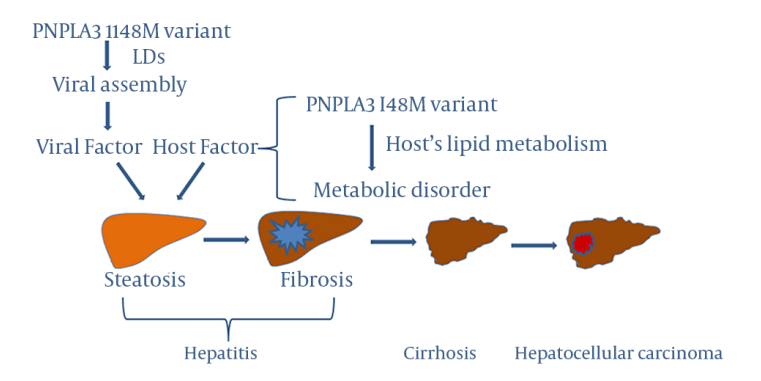

Figure 1. Role of the PNPLA3 I148M Variant in the Development and Progression of Liver Diseases in Chronic Hepatitis

\subsection{PNPLA3 I148M Variant and Autoimmune Liver} Diseases

Autoimmune liver diseases mainly include autoimmune hepatitis, Primary Sclerosing Cholangitis (PSC) and primary biliary cirrhosis (PBC) (47). They are cryptogenic liver diseases and recent articles reported the rare cases with them (48-50). Autoimmune hepatitis with biliary tract involvement is always seen in PSC and PBC $(51,52)$. Studies of the association of the PNPLA3 I148M variant with autoimmune liver diseases are scarce.

A recent study showed that (53) the PNPLA3 I148M variant affects the disease course of PSC and significantly decreased the actuarial survival rate free of liver transplantation in PSC patients with severe bile duct strictures. Interestingly, the study further demonstrated that the association between this variation and PSC followed a gender-specific pattern; the effect of I148M variant on actuarial survival was shown only in male, but female, PSC patients. This finding supports a previous report demonstrating a gender-specific pattern, suggesting a sexual dimorphism of the PNPLA3 I148M variant in NAFLD development (54). This preliminary study suggested that genetic testing for the PNPLA3 I148M variant is recommended due to the high risk of PNPLA3 I148M variant in PSC male patients, to improve diagnosis and subsequent therapies. Such an individualized decision-making and personalized medicine approach, including risk stratification, surveillance strategies and liver transplant allocation, may be an optimal choice of management, especially for male PSC patients. This study paved a new avenue for studying the role of the PNPLA3 I148M variant in autoimmune liver diseases; however, additional studies are needed to further elucidate the role of this variant in autoimmune liver diseases.

\subsection{PNPLA3 I148M Variant and Liver Transplanta- tion}

End-stage of liver disease is the most important indication for liver transplantation $(55,56)$ and the outcome of liver transplantation, especially its complications affects patients' quality of life (57). It is worthwhile to investigate the association of the PNPLA3 I148M variant with the outcome of liver transplantation.

$\mathrm{CHC}$ is the most common reason for liver transplantation in Western countries (58). In 2011, a preliminary study suggested that the PNPLA3 I148M variant in transplant recipients was not associated with recurrent $\mathrm{HCV}$ fibrosis progression (59). However, the study did not take the donors' PNPLA3 genotype into consideration. A recent study showed that the PNPLA3 I148M variant in the donors, rather than the recipients, was a risk predictor of poor post-Liver Transplant (LT) outcomes (i.e. increased risk of fibrosis progression, re-transplantation, or death) in $\mathrm{CHC}$ (60), indicating that liver rather than adipose tissue, was the exact site where this effect occurs and the donor genotype should also be a considerable factor 
for HCV-related liver transplantation. Thus, the ability to identify donors' genotype would help care providers assess the graft and establish a post-LT fibrosis progression management plan before and after transplantation, respectively.

Dunn et al. reported that (60) the PNPLA3 I148M variant in donors promoted fibrosis progression, but the group did not observe an association between the PNPLA3 I148M variant, neither of donors nor of recipients, with post-LT hepatic steatosis. However, in the study by Finkenstedt et al. (61), the PNPLA3 I148M variant of the recipients, rather than the donors, was associated with post-LT steatosis. The findings of these two studies indicate that PNPLA3mediated fibrosis progression and steatosis in HCV-related liver transplant patients might occur through separate pathways, although further investigation is needed to confirm the speculation.

Couto et al. suggested that the reason for higher post-LT metabolic syndrome and diabetes mellitus in Hispanic patients was associated with genetic differences caused by the PNPLA3 I148M variant (62). Another recent study showed that the recipient PNPLA3 I148M variant, rather than the donors' genotype, was independently associated with post-LT obesity; combined with the IL28B TT genotype, this variant was predictive of diabetes mellitus/ impaired fasting glucose (IFG) (63). A recent letter by Karlas et al. reported that (64) the PNPLA3 I148M variant of recipients was not only associated with post-LT steatosis, but also with post-LT diabetes mellitus. Lack of donor-related risks for post-LT metabolic disorders indicates that the peripherally mediated mechanism (via extrahepatic adipose tissue) may play a principal role in triglyceride accumulation regardless of adiponutrin activity in the graft liver (62-64). Taken together, these findings suggest that identification of patients with high risk for post-LT metabolic syndrome and diabetes mellitus before LT would help physicians make aggressive weight management plans in advance before and after operation.

\section{Results}

The PNPLA3 I148M variant is associated with chronic viral hepatitis, mainly via development of steatosis caused by host metabolic disorders. Since the I148M variant was associated with hepatocarcinogenesis in CHC patients, taking $1148 \mathrm{M}$ variant factor into consideration would assist in creating a reasonable therapeutic strategy to prevent future development of HCC in CHC patients.

There is a male gender-specific association between the PNPLA3 I148M variant and PSC with severe bile duct strictures. However, additional studies are needed to further elucidate the associations and the role of this variant in autoimmune liver diseases not only in PSC, but also in autoimmune hepatitis and primary biliary cirrhosis.

The associations of PNPLA3 I148M polymorphism in donors or recipients and post-LT outcomes are listed in Table 1. Generally, PNPLA3 I148M variant of the donors, rather than the recipients, is a risk predictor of poor postLT outcomes in HCV, including fibrosis progression or even a high re-transplant rate. In contrast, PNPLA3 I148M variant of the recipients, rather than the donors, is associated with post-LT metabolic disorders, including steatosis, obesity or diabetes mellitus/IFG. Recently, Dunn et al. reported that the PNPLA3 I148M variant did not increase post-LT mortality, despite the risk of this genotype for many severe liver diseases (60). However, further investigation is required to determine whether the PNPLA3 $148 \mathrm{M}$ variant is associated with prognosis of liver transplantation.

Table 1. Differential Associations of PNPLA3 I148M Polymorphism in Donors and Recipients With Post-Liver Transplant Outcomes

\begin{tabular}{lc}
\hline Associated Post-Liver Transplant Outcomes & References \\
\hline Donors & \\
$\begin{array}{l}\text { Fibrosis progression; Increased re-transplant } \\
\text { and survival rate }\end{array}$ & $(55)$ \\
$\begin{array}{l}\text { Recipients } \\
\text { Steatosis }\end{array}$ \\
$\begin{array}{l}\text { Obesity; diabetes mellitus; impaired fasting } \\
\text { glucose }\end{array}$ \\
\hline
\end{tabular}

\section{Conclusions}

Evidences have shown associations between I148M variant and chronic viral hepatitis, autoimmune liver diseases and the outcome of liver transplantation and these could provide clues for personalized therapies, especially for PSC male patients and in donor selection and prognosis estimation in liver transplantation. Additional studies are needed to further illustrate the mechanisms of I148M variant in chronic liver disease.

\section{Acknowledgements}

We would like to thank the Qingdao Municipal Hospital for their supports.

\section{Financial Disclosure}

Yong-Ning Xin, Man Jiang and Shi-Ying Xuan reported receiving research grants and honoraria from Qingdao Municipal Hospital.

\section{Funding/Support}

This review was supported by the National Natural Science Foundation of China (NSFC, No. 81170337 (http:/| www.nsfc.gov.cn/).

\section{References}

1. Romeo S, Kozlitina J, Xing C, Pertsemlidis A, Cox D, Pennacchio LA, et al. Genetic variation in PNPLA3 confers susceptibility to nonalcoholic fatty liver disease. Nat Genet. 2008;40(12):1461-5. 
2. Romeo S, Sentinelli F, Dash S, Yeo GS, Savage DB, Leonetti F, et al Morbid obesity exposes the association between PNPLA3 I148M (rs738409) and indices of hepatic injury in individuals of European descent. Int J Obes (Lond). 2010;34(1):190-4.

3. Valenti L, Alisi A, Galmozzi E, Bartuli A, Del Menico B, Alterio A, et al. I148M patatin-like phospholipase domain-containing 3 gene variant and severity of pediatric nonalcoholic fatty liver disease. Hepatology. 2010;52(4):1274-80.

4. Zain SM, Mohamed R, Mahadeva S, Cheah PL, Rampal S, Basu RC, et al. A multi-ethnic study of a PNPLA3 gene variant and its association with disease severity in non-alcoholic fatty liver disease. Hum Genet. 2012;131(7):1145-52.

5. Tian C, Stokowski RP, Kershenobich D, Ballinger DG, Hinds DA. Variant in PNPLA3 is associated with alcoholic liver disease. Nat Genet. 2010;42(1):21-3.

6. Graff M, North KE, Franceschini N, Reiner AP, Feitosa M, Carr JJ, et al. PNPLA3 gene-by-visceral adipose tissue volume interaction and the pathogenesis of fatty liver disease: the NHLBI family heart study. Int J Obes (Lond). 2013;37(3):432-8.

7. Valenti L, Dongiovanni P, Ginanni Corradini S, Burza MA, RomeoS. PNPLA3 I148M variant and hepatocellular carcinoma: a common genetic variant for a rare disease. Dig Liver Dis. 2013;45(8):619-24.

8. Hourigan LF, Macdonald GA, Purdie D, Whitehall VH, Shorthouse $\mathrm{C}$, Clouston A, et al. Fibrosis in chronic hepatitis $\mathrm{C}$ correlates significantly with body mass index and steatosis. Hepatology. 1999;29(4):1215-9.

9. Patton HM, Patel K, Behling C, Bylund D, Blatt LM, Vallee M, et al The impact of steatosis on disease progression and early and sustained treatment response in chronic hepatitis $C$ patients. $J$ Hepatol. 2004;40(3):484-90.

10. Poynard T, Ratziu V, McHutchison J, Manns M, Goodman Z, Zeuzem S, et al. Effect of treatment with peginterferon or interferon alfa-2b and ribavirin on steatosis in patients infected with hepatitis C. Hepatology. 2003;38(1):75-85.

11. Tanaka A, Uegaki S, Kurihara H, Aida K, Mikami M, Nagashima I, et al. Hepatic steatosis as a possible risk factor for the development of hepatocellular carcinoma after eradication of hepatitis $C$ virus with antiviral therapy in patients with chronic hepatitis C. World J Gastroenterol. 2007;13(39):5180-7.

12. Pekow JR, Bhan AK, Zheng H, Chung RT. Hepatic steatosis is associated with increased frequency of hepatocellular carcinoma in patients with hepatitis C-related cirrhosis. Cancer. 2007;109(12):2490-6.

13. Valenti L, Rumi M, Galmozzi E, Aghemo A, Del Menico B, De Nicola $S$, et al. Patatin-like phospholipase domain-containing 3 I148M polymorphism, steatosis, and liver damage in chronic hepatitis C. Hepatology. 2011;53(3):791-9.

14. Trepo E, Pradat P, Potthoff A, Momozawa Y, Quertinmont E, Gustot $\mathrm{T}$, et al. Impact of patatin-like phospholipase-3 (rs738409 $\mathrm{C}>\mathrm{G}$ ) polymorphism on fibrosis progression and steatosis in chronic hepatitis C. Hepatology. 2011;54(1):60-9.

15. Rueger S, Bochud PY, Dufour JF, Mullhaupt B, Semela D, Heim MH, et al. Impact of common risk factors of fibrosis progression in chronic hepatitis C. Gut. 2014

16. De Nicola S, Dongiovanni P, Aghemo A, Cheroni C, D'Ambrosio $\mathrm{R}$, Pedrazzini $\mathrm{M}$, et al. Interaction between PNPLA3 I148M variant and age at infection in determining fibrosis progression in chronic hepatitis C. PLoS One. 2014;9(8).

17. Cai T, Dufour JF, Muellhaupt B, Gerlach T, Heim M, Moradpour $D$, et al. Viral genotype-specific role of PNPLA3, PPARG, MTTP, and IL28B in hepatitis C virus-associated steatosis. $J$ Hepatol. 2011;55(3):529-35.

18. Rembeck K, Maglio C, Lagging M, Christensen PB, Farkkila M Langeland N, et al. PNPLA 3 I148M genetic variant associates with insulin resistance and baseline viral load in HCV genotype 2 but not in genotype 3 infection. BMC Med Genet. 2012;13:82.

19. Zampino R, Coppola N, Cirillo G, Boemio A, Pisaturo M, Marrone A, et al. Abdominal fat interacts with PNPLA3 I148M, but not with the APOC3 variant in the pathogenesis of liver steatosis in chronic hepatitis C. J Viral Hepat. 2013;20(8):517-23.

20. Despres JP, Lemieux I. Abdominal obesity and metabolic syndrome. Nature. 2006;444(7121):881-7.
21. Valenti L, Aghemo A, Stattermayer AF, Maggioni P, De Nicola S, Motta BM, et al. Implications of PNPLA3 polymorphism in chronic hepatitis $C$ patients receiving peginterferon plus ribavirin. Aliment Pharmacol Ther. 2012;35(12):1434-42.

22. Clark PJ, Thompson AJ, Zhu Q, Vock DM, Zhu M, Patel K, et al. The association of genetic variants with hepatic steatosis in patients with genotype 1 chronic hepatitis C infection. Dig Dis Sci. 2012;57(8):2213-21.

23. Moritou Y, Ikeda F, Iwasaki Y, Baba N, Takaguchi K, Senoh T, et al. Impact of comorbid hepatic steatosis on treatment of chronic hepatitis $C$ in Japanese patients and the relationship with genetic polymorphism of IL28B, PNPLA3 and LDL receptor. Acta Med Okayama. 2014;68(1):17-22.

24. Aghemo A. Understanding the role of PNPLA3 genetic variants in patients with chronic hepatitis C infection. Dig Dis Sci. 2012;57(8):1977-9.

25. Corradini SG, Burza MA, Molinaro A, Romeo S. Patatin-like phospholipase domain containing 3 sequence variant and hepatocellular carcinoma. Hepatology. 2011;53(5):1776.

26. Nischalke HD, Berger C, Luda C, Berg T, Muller T, Grunhage F, et al. The PNPLA3 rs738409148M/M genotype is a risk factor for liver cancer in alcoholic cirrhosis but shows no or weak association in hepatitis C cirrhosis. PLoS One. 2011;6(11).

27. Guyot E, Sutton A, Rufat P, Laguillier C, Mansouri A, Moreau R, et al. PNPLA3 rs738409, hepatocellular carcinoma occurrence and risk model prediction in patients with cirrhosis. $J$ Hepatol. 2013;58(2):312-8.

28. Sato M, Kato N, Tateishi R, Muroyama R, Kowatari N, Li W, et al. Impact of PNPLA3 polymorphisms on the development of hepatocellular carcinoma in patients with chronic hepatitis $C$ virus infection. Hepatol Res. 2014;44(10):E137-44.

29. Thomopoulos KC, Arvaniti V, Tsamantas AC, Dimitropoulou D, Gogos CA, Siagris D, et al. Prevalence of liver steatosis in patients with chronic hepatitis B: a study of associated factors and of relationship with fibrosis. Eur J Gastroenterol Hepatol. 2006;18(3):233-7.

30. Petta S, Camma C, Di Marco V, Macaluso FS, Maida M, Pizzolanti G, et al. Hepatic steatosis and insulin resistance are associated with severe fibrosis in patients with chronic hepatitis caused by HBV or HCV infection. Liver Int. 2011;31(4):507-15.

31. Wong VW, Wong GL, Yu J, Choi PC, Chan AW, Chan HY, et al. Interaction of adipokines and hepatitis B virus on histological liver injury in the Chinese. Am J Gastroenterol. 2010;105(1):132-8.

32. Vigano M, Valenti L, Lampertico P, Facchetti F, Motta BM, D'Ambrosio R, et al. Patatin-like phospholipase domain-containing 3 I148M affects liver steatosis in patients with chronic hepatitis B. Hepatology. 2013;58(4):1245-52.

33. Falleti E, Fabris C, Cmet S, Cussigh A, Bitetto D, Fontanini E, et al. PNPLA3 rs738409C/G polymorphism in cirrhosis: relationship with the aetiology of liver disease and hepatocellular carcinoma occurrence. Liver Int. 2011;31(8):1137-43.

34. Tong J, Guo J, Hu J, Hou S, Zhang Y, Li Q. Correlation between patatin-like phospholipase domain-containing protein 3 gene polymorphisms and liver cirrhosis in a chinese han population with chronic hepatitis B. Hepat Mon. 2014;14(8).

35. Negro F, Sanyal AJ. Hepatitis C virus, steatosis and lipid abnormalities: clinical and pathogenic data. Liver Int. 2009;29 Suppl 2:26-37.

36. Hui JM, Kench J, Farrell GC, Lin R, Samarasinghe D, Liddle C, et al. Genotype-specific mechanisms for hepatic steatosis in chronic hepatitis C infection. J Gastroenterol Hepatol. 2002;17(8):873-81.

37. Fartoux L, Poujol-Robert A, Guechot J, Wendum D, Poupon R, Serfaty L. Insulin resistance is a cause of steatosis and fibrosis progression in chronic hepatitis C. Gut. 2005;54(7):1003-8.

38. Aly HH, Oshiumi H, Shime H, Matsumoto M, Wakita T, Shimotohno K, et al. Development of mouse hepatocyte lines permissive for hepatitis C virus (HCV). PLoS One. 2011;6(6).

39. Bassendine MF, Sheridan DA, Felmlee DJ, Bridge SH, Toms GL, Neely RD. HCV and the hepatic lipid pathway as a potential treatment target. J Hepatol. 2011;55(6):1428-40.

40. Targett-Adams P, Boulant S, Douglas MW, McLauchlan J. Lipid metabolism and HCV infection. Viruses. 2010;2(5):1195-217. 
41. Olofsson SO, Bostrom P, Andersson L, Rutberg M, Levin M, Perman J, et al. Triglyceride containing lipid droplets and lipid droplet-associated proteins. Curr Opin Lipidol. 2008;19(5):441-7.

42. McLauchlan J. Lipid droplets and hepatitis $C$ virus infection. Biochim Biophys Acta. 2009;1791(6):552-9.

43. Moriya K, Yotsuyanagi H, Shintani Y, Fujie H, Ishibashi K, Matsuura Y, et al. Hepatitis $\mathrm{C}$ virus core protein induces hepatic steatosis in transgenic mice. J Gen Virol. 1997;78 ( Pt 7):1527-31.

44. Dyson JK, Waller J, Turley A, Michael E, Moses S, Valappil M, et al Hepatitis B in pregnancy. Frontline Gastroenterol. 2014;5(2):111-7.

45. Chamoun Z, Vacca F, Parton RG, Gruenberg J. PNPLA3/adiponutrin functions in lipid droplet formation. Biol Cell. 2013;105(5):219-33.

46. Bugianesi E, Salamone F, Negro F. The interaction of metabolic factors with HCV infection: does it matter? J Hepatol. 2012;56 Suppl 1:S56-65.

47. Xia HHX. Liver Diseases and Autoimmunity.Journal of Clinical and Translational Hepatology. 2013;1(2):144

48. Deutsch M, Emmanuel T, Koskinas J. Autoimmune Hepatitis or Wilson's Disease, a Clinical Dilemma. Hepat Mon. 2013;13(5).

49. Geramizadeh B, Nikeghbalian S, Shamsaifar A, Kazemi K, Malekhosseini SA. Hepatocellular carcinoma in two patients with autoimmune hepatitis, a single center experience and review of the literature. Hepat Mon. 2013;13(4).

50. Rezaee Zavareh MS, Alavian SM, Karimisari H, Shafiei M, Saiedi Hosseini SY. Occult hepatitis C virus infection in patients with autoimmune hepatitis. Hepat Mon. 2014;14(8)

51. Pratico AD, Salafia S, Barone P, La Rosa M, Leonardi S. Type II Autoimmune Hepatitis and Small Duct Sclerosing Cholangitis in a Seven Years Old Child: An Overlap Syndrome? Hepat Mon. 2013;13(12).

52. Najafi M, Sadjadei N, Etekhari K, Khodadad A, Motamed F, Fallahi GH, et al. Prevalence of Celiac Disease in Children with Autoimmune Hepatitis and vice versa. Iranian Journal of Pediatrics. 2014;24(6):723-8

53. Friedrich K, Rupp C, Hov JR, Steinebrunner N, Weiss KH, Stiehl A, et al. A frequent PNPLA3 variant is a sex specific disease modifier in PSC patients with bile duct stenosis. PLoS One. 2013;8(3).
54. Sookoian S, Pirola CJ. Meta-analysis of the influence of I148M variant of patatin-like phospholipase domain containing 3 gene (PNPLA3) on the susceptibility and histological severity of nonalcoholic fatty liver disease. Hepatology. 2011;53(6):1883-94.

55. Murray KF, Carithers RLJ, Aasld . AASLD practice guidelines: Evaluation of the patient for liver transplantation. Hepatology. 2005;41(6):1407-32.

56. Dehghani SM, Haghighat M, Imanieh MH, Honar N, Negarestani AM, Malekpour A, et al. Autoimmune hepatitis in children: experiences in a tertiary center. Iran J Pediatr. 2013;23(3):302-8.

57. Lankarani KB. Liver Transplantation for Quality as well as Quantity of Life. Hepat Mon. 2013;13(10)

58. Akamatsu N, Sugawara Y. Liver transplantation and hepatitis C. Int J Hepatol. 2012;2012:686135.

59. Do ON, Eurich D, Trautwein C, Neuhaus P, Neumann UP, Wasmuth HE. The common I148 M variant of PNPLA3 does not predict fibrosis progression after liver transplantation for hepatitis C. Hepatology. 2011;54(4):1483-4.

60. Dunn W, O'Neil M, Zhao J, Wu CH, Roberts B, Chakraborty S, et al Donor PNPLA3 rs738409 genotype affects fibrosis progression in liver transplantation for hepatitis C. Hepatology. 2014;59(2):453-60.

61. Finkenstedt A, Auer C, Glodny B, Posch U, Steitzer H, Lanzer G, et al. Patatin-like phospholipase domain-containing protein 3 rs738409-G in recipients of liver transplants is a risk factor for graft steatosis. Clin Gastroenterol Hepatol. 2013;11(12):1667-72.

62. Couto CA, Gelape CL, Doycheva IB, Kish JK, Martin P, Levy C. Ethnicity predicts metabolic syndrome after liver transplant. Hepatology International. 2013;7(2):741-8.

63. Watt KD, Dierkhising R, Fan C, Heimbach JK, Tillman H, Goldstein $\mathrm{D}$, et al. Investigation of PNPLA3 and IL28B genotypes on diabetes and obesity after liver transplantation: insight into mechanisms of disease. Am J Transplant. 2013;13(9):2450-7.

64. Karlas T, Kollmeier J, Böhm S, Müller J, Tröltzsch M, Weimann A, et al. P805 Non-Invasive Characterization of Post-Transplant Fatty Liver Disease Reveals High Prevalence of Graft Injury in Association with Metabolic Syndrome, Recipient Pnpla3 Genotype and Alcoholic Liver Cirrhosis. Journal of Hepatology. 2014;60(1):S339. 